\title{
Comunidades virtuales, nuevos ambientes mismas inquietudes: el caso de Taringa!
}

\author{
David Ramírez Plascencia \\ Universidad de Guadalajara,Guadalajara, México. Email: david.ramirez@redudg.udg.mx \\ José Antonio Amaro López \\ Universidad de Guadalajara, Guadalajara, México. Email: josea@csh.udg.mx
}

\begin{abstract}
Resumen: Las comunidades en línea han sido vistas como una nueva esfera social que coadyuva al surgimiento de procesos democráticos de forma más rápida y óptima, de lo que sucede en las relaciones del mundo físico. Sin embargo, los alcances y características de estas interacciones sociales aún están por verse. El propósito de este documento es discutir hasta qué punto la acción de los individuos en estas comunidades difiere del funcionamiento tradicional de cualquier comunidad ¿Hasta dónde los espacios virtuales cumplen con las aspiraciones y promesas con las que han sido investidos? Un primer punto versará sobre las complejidades del establecimiento de comunidades en línea, para después pasar a determinar la forma en que se establecen las normas en estos espacios. Por último el trabajo se enfocará al estudio de caso de una comunidad virtual argentina denominada Taringa!
\end{abstract}

Palabras Clave: Comunidades Virtuales, Cibercultura, Democracia.

\section{Virtual communities, new environments same concerns: the case of Taringa!}

\begin{abstract}
Online communities have been seen as a new social sphere that contributes to the emergence of democratic processes faster and better than those in the physical world. However, the scope and characteristics of these virtual social interactions needs a more profuse and deeper analysis. The purpose of this paper is to discuss how the actions of individuals in these communities differ from those in the traditional community. Do these virtual spaces accomplish the aspirations and promises we have invest in them? One point will address the complexities of establishing online communities, then moving on to determine how do norms are set in these virtual spaces. Finally the work will focus on the case study of a virtual community in Argentina called Taringa!
\end{abstract}

Key Works: Virtual Communities, Cyberculture, Democracy.

\section{As comunidades virtuais, ambientes novos mesmas preocupações: o caso do Taringa!}

Resumo: As comunidades online têm sido vistas como uma nova esfera social que contribui para o surgimento de processos democráticos mais rapidamente 
e de forma otimizada, do que acontece nas relações do mundo físico. No entanto, o alcance e as características dessas interações sociais continuam a ser visto. $\mathrm{O}$ objetivo deste artigo é discutir como as ações dos indivíduos nestas comunidades diferem do funcionamento tradicional de qualquer comunidade como espaços virtuais dónde satisfazer as aspirações e promessas que foram investidos? Um ponto vai abordar as complexidades da criação de comunidades on-line, passando em seguida para determinar como definir os padrões nestas áreas. Finalmente, o trabalho se concentrará no estudo de caso de uma comunidade virtual argentina chamada Taringa!

Palavras-chave: Comunidades Virtuais, Democracia, Cibercultura.

$$
* * *
$$

\section{Introducción}

Con el surgimiento de espacios para socializar en línea como las redes sociales, es cada vez más complicado poder delimitar el alcance de las relaciones tanto en la virtualidad como en la vida fuera de las computadoras, así comoestablecer donde empiezan y terminan los vínculos afectivos entre los individuos que se mueven de manera constante entre estos dos mundos. Si anteriormente, la palabra comunidad hacía alusión al ensamblaje social de seres que cohabitan en un espacio pequeño bien definido, ahora cuando se habla de relaciones virtuales,ya no es tan claro que se entiende cuando se hace alusión a la palabra comunidad (Palloff, y PrattKeith: 28). En la búsqueda de comprender estas nuevas relaciones de los individuos con la tecnología, el uso de conceptos tradicionales tiene una función determinante, pero a la vez conflictiva si se aplica a las comunidades virtuales, porque las realidades que engloban no siempre corresponden a los fenómenos virtuales que se trata de incorporar.

La primera mención del término comunidades virtuales aparece en la obra de Rheingold, The Virtual Community: Homesteading on the Electronic Frontier (1993: 5), en la cual se definen estos espacios como "agregados sociales que emergen desde Internet, y cuya particularidad recae en el hecho de portar discusiones públicas, con suficiente sentido humano para crear relaciones sociales en el ciberespacio”. Desde entonces el término ha estado sujeto a controversia y crítica; así, para Fernback y Thompson (1995) una comunidad virtual es más indicativa de un conjunto de personas tratando de consolidar un espacio en línea, que de una comunidad en el sentido más tradicional de la expresión. Es decir, estos intentos de comunidades virtuales no pueden consolidarse como tales, porque carecen delsentido de pertenencia que se deriva de un trato habitual cara-cara de las relaciones físicas.

Sin embargo, la idea de que una comunidad deba basarse en interacciones cara-cara o físicas, ha sido rebatida hace mucho por autores como Anderson (1991) que proponen que las comunidades también pueden basarse en nexos como el nacionalismo, que facilitan la integración de individuos que no tienen contacto directo de manera cotidiana, y que sin 
embargo comparten un conjunto de significaciones que los identifican como pertenecientes a un mismo grupo. De ahí que espacios como Taringa!puedan ser tratados de la misma manera que una comunidad tradicional, porque los contactos en línea pueden generar verdaderas redes de individuos que comparten sentimientos, intereses y objetivos comunes. Aunque, por otro lado, no se puede pasar por alto el hecho de que las comunidades virtuales no pueden vivir aisladas y separadas del soporte del mundo material.

Por lo anterior, las comunidades en línea representan una extensión del mundo real, y si bien se constituyen en un medio intangible, se fortalecen a través de las mismas relaciones sociales que se pueden observar en el mundo físico. Por tal razón, las observaciones de este ensayo discrepan de manera absoluta con aquellos puntos de vista que consideran al ciberespacio, y otros medios virtuales, como espacios totalmente diferentes del mundo social-físico, y por lo tanto revisten otro tipo de relaciones sociales, diferentes de aquellas que usualmente se han establecido entre los seres humanos a lo largo de la historia.

Lejos de ser el ciberespacio un punto y parte de los estudios de la cultura, se consolida más bien como una nueva área para estudiar fenómenos tradicionales en el ámbito de la antropología. De ahí que este artículo utilice recursos metodológicos tradicionales como la Descripción Densa de Clifford Geertz, sin considerar que las comunidades virtuales deban de ser estudiadas como unidades autónomas e independientes, necesitadas de otra clase de enfoques.

El presente trabajo analiza una comunidad virtual argentina, Taringa!, con alcances internacionales, donde se han establecido patrones muy claros de comportamiento y estructuras de vigilancia efectivas para controlar el rol de cada miembro. Lo cual representa un claro ejemplo de cómo las comunidades en línea, por muy libertarias que pretendan ser, necesitan forzosamente para existir, de mecanismos de represión eficaces para controlar a sususuarios. Las comunidades virtuales son construcciones que parten de preconcepciones establecidas: términos como comunidad, miembros, moderador,normas de conducta, se adaptan a un nuevo entorno, pero no varían de manera determinante. El ser humano crea nuevas comunidades de la misma manera en que lo ha hecho siempre, el ciberespacio no es una excepción. Las comunas virtuales reflejan la necesidad natural del ser humano por estrechar lazos solidarios con sus semejantes.

\section{Internet $\dot{¿}$ Un nuevo comienzo?}

Una Ciudad digital no sólo es el diseño binario de un espacio de convivencia. También revela de manera ineludiblemente anhelos, sueños y promesas que han estado presentes en las sociedades occidentales durante siglos. El ciberespacio representó, y aún representa para muchos, una nueva tierra prometida. 
Existe una tendencia general en pensar que el ciberespacio puede ser la plataformaidónea para la creación de una ciudad digital ideal. Un entorno que asemeje un hábitat común y corriente en su diseño, pero que contenga todas las mejoras, beneficios y adelantos a los que no se puede acceder en el mundo físico. De esta manera, ya no se busca una ciudad digital, sino más bien un topusuranus ${ }^{1}$ donde todos nuestros anhelos se puedan conformar a la perfección.

Sin embargo, desarrollar una imagen de ciudad ideal en la era de la saturación digital parece una empresa complicada. Por muchos esfuerzos que se hayan hecho por desmaterializar o desaparecer el concepto tradicional de ciudad dentro del ciberespacio, seguimos anclados de manera inexorable al mismo (Gibson 1984, citado por Boyer 1996: 138).El novelista William Gibson (1984), escritor que utilizó por primera vez el término ciberespacio, señala "que este espacio virtual corresponde a una ciudad que no tiene una forma imaginable o una frontera definida". Si bien la palabra ciberespacio se utilizó por primera vez dentro de una novela de ciencia ficción. Lo cierto es que dicha descripción de Gibson ha servido como base para futuras conceptualizaciones del espacio virtual de Internet o ciberespacio, tanto en ámbitos legales estadounidenses como en foros de discusión en Internet.

El ciberespacio de Gibson es un mundo diferente basado en un concepto culturalmente creado: La ciudad. En efecto, para poder describir un espacio tan complejo y cambiante como el ciberespacio,tanto en el caso de un novelista como para el Gobierno de Estados Unidos, en sus constantes peleas legales por censurar Internet ${ }^{2}$, no hay otra manera que apelar a conceptos ya conocidos y asimilados por una colectividad. De otra forma no sería posible explicar que cosa es el ciberespacio.

Así para ilustrar un lugar sin forma ni frontera definida, se apela a uno que si lo tiene. Una ciudad, desde el punto de vista de la sociedad occidental, si es un lugar definido formalmente y con límites. Lo que se puede apreciar en resumidas cuentas es que por mucho que se pueda apelar de que las comunidades en línea son diferentes a aquellas del mundo físico. Lo cierto es que incluso, en espacios tan laxos como el virtual, el uso de términos como delimitación y forma son tan necesarios como en la vida real. Porque el ser humano, culturalmente hablando, necesita darle un sentido a las cosas para poderlas interpretar, así las comunidades virtuales sólo pueden ser construidas con base a categorías culturalmente aceptadas.

Se ha argüidocon insistencia que Internet ha modificado nuestras concepciones de espacio y tiempo, que hemos ido perdiendo las barreras, los límites y las formas, y que poco sabemos dónde queda un territorio y empieza otro (Boyer 1996: 19).Esta afirmación, tiene parte de razón: el ciberespacio traspasa las barreras nacionales y también minimiza los efectos del tiempo, porque permite la comunicación asincrónica entre los usuarios. Pero también es cierto que la única forma en que se le puede dar sentido a un espacio tan amplio y complejo como el virtual, es através de para- 
dójicamente, delimitaciones espaciales tradicionales, usadas de manera regular en la vida cotidiana.

De esta forma tenemos: Sitios personales, espacios personales, blog personales, es decir, por muy libre que sea la categorización del ciberespacio, existe la necesidad de establecer límites muy claros entre la propiedad de un usuario y de otro, entre una red y otra. Y si bien los Estados-nación no pueden hacerse de todo el control en Internet, no por ello los términos frontera, jurisdicción y propiedad han dejado de ser útiles en estas ciberciudades. La frontera entre lo tuyo y lo mío, es tan importante en el ciberespacio como lo era desde los primeros albores de humanidad.

\section{¿Es tan diferente el ciberespacio al mundo real?}

El ciberespacio es considerado como una realidad virtual paralela al mundo físico (Grosz 2001)¿Pero realmente es tan diferente? ¿ Será tan utópico este lugar? Es decir, un lugar sin forma ni límites. Pero aún más importante ¿Será posible fundar una nueva ciudad virtual desde cero, descartando los modelos represivos de las ciudades físicas? ${ }^{3}$

Si bien es cierto que en el ciberespacio se han modificado las relaciones afectivas, económicas y educativas,estas nunca han dejado de ser las mismas que se buscan en el mundo real: Educación a distancia, es una extensión de las instituciones educativas, y el comercio en línea es el mismo contrato de compra-venta acuñado en el derecho romano. Lejos de considerar ese mundo virtual diferente, es una extensión de las relaciones del mundo físico. Es un nuevo medio para perseguir los mismos fines. Es un campo donde se siembran las mismas instituciones sociales.

En 1996, John Perry Barlow, redactó la famosa declaración de Independencia del Ciberespacio, dondedestacaba el hecho de que: «Empresas y gobierno no tenían soberanía en estos nuevos espacios». También afirmaba que estas «empresas y autoridades desconocían su cultura, su ética y códigos de conducta». Ellos, los cibernautas, según Barlow, "Estaban formando su propio contrato social» que será regido «por otras propiedades, expresiones, identidades, movimientos...”Visto a más de dos décadas de distancia este discurso continúa siendo atrayente: formar una nueva utopía. Un mundo mejor para todos.

Sin embargo, qué tanto de razón puede tener todo este discurso cuando se contrasta con la realidad de las relaciones sociales en Internet $\dot{i}$ En verdad es posible construir una sociedad sin límites territoriales y jurisdiccionales? ¿Se puede hacer a un lado la ética, los códigos y todas las instituciones tradicionales, y empezar desde cero?

Con respecto al cuestionamiento sobre la imposibilidad de controlar el tráfico en línea, un estudio minucioso de las redes en Internet nos permite entender que saltarse dicho control es imposible. La conectividad 
en Internet se basaen un entramado tecnológico donde interactúan entre sí los nombres de dominio ${ }^{4}$, las direcciones $I P^{5}$, y los backbones. ${ }^{6}$ Todos estos elementos hacen alusión a una ubicación concreta, pero sobre todo, a un control sobre los flujos de datos.

Pero incluso, si fuera posible saltarse todos estos controles ¿Se puede afirmar que los cibernautas hubieran podido crear un ciberespacio basado en relaciones diferentes a las que cotidianamente sostienen en su sociedad, utilizando una nueva lógica, otra ética, y otro sentido de la vida social? Los cibernautas, empero, no van a otro mundo, sino que expanden en el ciberespacio el que ya tienen. Ejemplos como Second Life ${ }^{7}$, revelan como los usuarios buscan no un espacio estructuralmente diferente, sino más bienposibilidades diferentes a las disponibles en su mundo material.

Lo natural, o lo que llamamos natural, se consolida como la unión entre el medio en que vivimos y el complejo cultural que construimos con base enéste. De ahí que la conquista de nuevos espacios como la realidad virtual nos remitan a la incorporación de nuestros complejos culturales. De esta manera redefinimos estos lugares bajo una categoría de símbolospertenecientes a un complejo cultural compartido. El ciberespacio es un campo nuevo donde se plantan las mismas semillas que se conocen, y se trata de cosechar las mismas relaciones sociales.El ciberespacio visto como una reincorporación, nos permite entender cómo es posible conocer a los cibernautas a través del ambiente que han creado. Porque, lo natural, ya sea biológico, físico o tecnológico siempre se convierte en un medio que expone la identidad de sus creadores (Giannachi 2004: 69).

\section{e-Tribus y comunidades en línea}

Grandes esperanzas se han volcado sobre Internet y su capacidad para favorecer la integración de los individuos en comunidades. Sin embargo, no hay un acuerdo generalizado sobre los elementos esenciales que deben existir para considerar un espacio en línea como una comunidad virtual (Bell 2001: 92). ¿Qué es lo que hace que una comunidad sea considerada como tal? Sin entrar en discusiones sobre sí la presencialidad es un factor de peso para la conformación de una agrupación en línea, sobre sí las reuniones cara-cara son totalmente indispensables para que una comunidad pueda llamarse así, o sobre sí se tratan o no de espacios colectivizados donde cada individuo se consolida como un miembro y desempeña una función específica. Dejando a un lado esta discusión, nos enfocaremos a interpretar las acciones de losmiembros de estos espacios, y comprenderque representa para ellos el interactuar con otras personas en línea. En pocas palabras, serán los propios cibernautas quienes dirán si pertenecen o no a una comunidad virtual.

En este sentido es importante para nuestro estudio los aportes de Cliffort Geertz (1973) sobre el papel de cada individuo -en este caso, de cada cibernauta- en la construcción de sus propios significados. El trabajo 
del antropólogo "virtual” o el estudioso de la cibercultura no es hacer una mera descripción de hechos, sino más bien,el adentrarse en la descripción densa o compleja sobre el significado de los actos que un miembro de una comunidad en línea tiene que realizar para adecuarse a la manera de ser de los demás integrantes. (Geertz 1973: 11).

De esta manera, se deja que el propio miembro de espacios como Yahoo! Answers, e-Bay, Facebook, Taringa! manifieste si sus acciones durante su estancia en estos servicios son parte de una lógica comunal o no. Bajo este enfoque, no se busca asentar que los actos de los cibernautas puedan caber dentro de un espectro comunitario según tal o cual visión teórica, sino más bien se afirma que dichas acciones son comunitarias porque quienes los realizan, las consideran como tales (Castells 2004: 73).

Si bien el concepto de comunidad se formó atendiendo a una lógica diferente, una lógica física, no virtual. No hay, sin embargo,una oposicióntan marcada que nos permita decir que las comunidades virtuales y físicas son totalmente diferentes. Un examen minucioso de las acciones de estos sujetos nos revelará que efectivamente ellos: juegan, compran, platican, comparten, discuten, roban, se enamoran de la misma manera que en el mundo real. Claro que el medio digital impone diferencias, pero estas diferencias no representan un cambio trascendental en las relaciones humanas. Todos estos asuntos sólo tienen su razón de ser en la colectividad, porque lejos de ésta pierden su significado. De ahí que un medio digital sólo facilite ésta interacción pero no la transforma totalmente. Los usuarios virtuales construyen, tejen y elaboran sus relaciones de la única forma en que saben hacerlo, y construyen comunidades virtuales, porque sólo en estos lugares pueden crecer y fortalecerse dichas relaciones.

Las comunidades virtuales o en línea, se asemejan con mucho a tribus, grupos identificados como únicos, que siempre tratan de diferenciarse. Las tribus o comunidades virtuales son autónomas y homogéneas con un lenguaje, una cultura y un espacio delimitado y común a sus miembros. Las e-tribus representan el resurgimiento de esta antigua forma de agrupación, que en el ciberespacio ha tomado un segundo aíre (Adams 2008: VIII).

\section{Las e-tribus y las normas...}

Las e-tribus o comunidades en línea, utilizan normas que son pautas de comportamiento ya creadas y establecidas en el mundo físico. Son adecuaciones de comportamientos de la vida cotidiana: intolerancia hacia cierto tipo de individuos, prohibición de ciertos contenidos, y la imposición de una serie de ritos, requisitos y demás pruebas de aceptación (Adams 2008: X).

Toda comunidad virtual establece sus propias normas ${ }^{8}$, que con el 
tiempo se consolidad y crean instituciones, es decir, las reglas del juego, los constreñimientos u obligaciones creadas que le dan forma a la interacción social. Las instituciones determinan su validez a través de la fuerza coercitiva que ejercen sobre sus miembros. Donde hay coerción, hay institución. Es el concepto de institución un elemento determinante quenos ayuda a entender el proceso mediante el cual los miembros de una e-tribu asimilan las normas y los patrones de conducta en una comunidad dada.

Dentro de las normas de una e-tribu existen dos tipos de normas: por un lado, las que establecen constreñimientos de carácter informal, prácticas sociales provenientes de una información socialmente transmitida y que forman parte de la herencia que llamamos cultura y en el otro extremo, las reglas formales, jerárquicamente ordenadas, que constituyen el mundo del derecho positivo.

En el caso de Internet, su cuerpo normativo está compuesto por normas de carácter estatal: organizaciones internacionales y gobierno estatales. Pero también por disposiciones de origen privado: empresas y usuarios particulares. En un ambiente tan laxo, legalmente hablando, como es el ciberespacio, donde las normas de un Estado no siempre se cumplen, la única forma de explicar cómo se ha consolidado un orden virtual en las comunidades virtuales es apelando a las instituciones como elementos de cohesión y coerción. Dichas instituciones, sin embargo, entremezclan elementos tanto de las normas estatales como de aquéllos usos privados.

El proceso de control en las comunidades en línea, es en muchos aspectos, un proceso de consolidación de instituciones. En este caso, el fortalecimiento de las instituciones depende por mucho de los administradores de contenidos o moderadores,pues de ellos depende vigilar la conducta de todos los miembros. Es importante señalar que cada colectividad virtual reviste un patrón especial de conducta. Ya se trate de una decisión del administrador del sitio o una norma legal estatal positiva,el proceso de control, como el proceso de creación de una comunidad virtual, implicará siempre una reincorporación de elementos ya aprendidos hacia nuevos espacios.

Para explicar el proceso de institucionalización del ciberespacio, es decir la forma mediante la cual se forman las instituciones en las comunidades virtuales, es necesario remitirse primeramentea la cualidad del ser humano de asimilar nuevas experiencias en su entorno, es decir, la plasticidad (Luckmann y Berger 1995: 68). Este término hace alusión a la capacidad del ser humano para absorber como una esponja las circunstancias que lo rodean: lengua, religión, posición social. Esta característica es fundamental para entender el proceso de socialización en una comunidad en Internet, porque los miembros no están habituados a los mismos usos y costumbres.

Gracias a la plasticidad, estos cibernautasse han cohesionado en comunidades electrónicas, en las cuales las diferencias de uso han sido 
reemplazadas por nuevas tipificaciones reciprocas de acciones. La manera en que dicho proceso ha llegado a producirse asemeja con mucho al modelo de una tribu de cazadores donde los más ancianos —en este caso los usuarios más experimentados_- enseñan a los recién llegados las técnicas de caza, en nuestro caso las formas apropiadas de comportamiento (Burk 1999: 6).

A este sistema de usos aceptados dentro de una colectividad virtual se le suele denominar nettiqueta (Matías 1998: 18). Bajo este nombre se hace patente un conjunto muy amplio de disposiciones que permiten crear un cierto nivel de armonía entre los usuarios. Así por ejemplo, los miembros recientes de la comunidad aprendena abstenerse de enviar correo basura al servicio de mensajería electrónica de otro miembro, o evitan usar palabras consideradas como ofensivas dentro de dicha comunidad. De los argumentos antes expresados, surge una interrogación: ¿cómo se establecen las reglas del juego en un lugar donde los actores pueden pertenecer a niveles económicos, sociales, culturales y nacionales tan diferentes? Para responder a esta interrogante debemos partir del hecho de que toda comunidad desde su origen ya está normada y por lo tanto tiene sus propios usos (Lessig 2001: 51).

Cada comunidad que se conformaen el ciberespacio cuentan con sus propios valores, usos y por lo tanto sus propias instituciones. Estas instituciones, por el simple hecho de existir, controlan el comportamiento de los nuevos integrantes de la comunidad, estableciendo pautas definidas de antemano que lo canalizan a una dirección determinada, en oposición a las nuevas ideas que dicho integrante traería consigo (Luckmann 1995 y Berger 1995:76).

Cuando este nuevo elemento se integra a la colectividad, está obligado a adoptar un comportamiento razonable dentro de la misma, de lo contrario se haría acreedor a una sanción, o en muchos casos la expulsión del colectivo. Las instituciones, en las comunidades virtuales, son experimentadas por los nuevos integrantes como si poseyeran una realidad propia que se presenta al individuo como un hecho extremo y coactivo. De esta forma se garantiza la uniformidad de comportamiento.

Todo uso institucionalizado, sin embargo, necesita de la creación de un cuerpo de conocimientos para ser transmitido. Un guión que facilite el comportamiento institucionalmente apropiado. Es ahí donde el papel de los roles tiene un lugar preponderante para lograr la cohesión de la comunidad virtual. Por ejemplo, la figura de un policía encargado de vigilar la conducta de los integrantes y castigar a los infractores. Es sólo mediante la creación de roles y actoresque el comportamiento de la comunidad se vuelve susceptible de coacción, y por lo tanto las instituciones permanecen.

Porque lejos de ser la virtualización un proceso separado y ajeno a 
la cultura, se muestra como una extensión de las instituciones de los individuos. La consolidación de las comunidades en línea es un proceso de reproducción de prácticas y estructuras culturales, como las de poder (Fuchs 2008: 300).

Las comunidades virtuales son espacios donde la gente puede construir nuevas redes sociales a partir de sus significados ya conocidos. Dentro de las comunidades virtuales se crean redes de significado bajo las cuales los miembros pueden interactuar unos a otros. Las primeras comunidades virtuales datan de principios de la década de los 80s.

Un ejemplo de este tipo de concreciones es el proyecto WELL (www.well.com). Desarrollado a mediados de la década de los 80s en la zona de San Francisco California, ha tenido desde su creación un especial interés por desarrollar debates virtuales sobre temas políticos, culturales y sociales. Muchos periodistas cuentan con una cuenta gratuita para accesar. Con el tiempo la comunidad derivo en encuentros presenciales, fortaleciendo los lazos afectivos entre los miembros.

Las comunidades virtuales pueden crecer de manera exponencial y albergar decenas de millones de miembros, pero incluso hay comunidades virtuales que pueden congregar literalmente a toda una ciudad. El proyecto Blacksburg Electronic Village (bev.net), fue desarrollado por el Instituto Tecnológico de Virginia (Virginia Polytechnic Institute), y la Universidad del Estatal de Virginia (Virginia Tech). Su aplicación ha facilitado la conexión e integración de 36, 000 personas del Condado de Blacksburg (Equivalente al 90\% de la población total).

Una comunidad virtual se desarrolla en Internet, pero también puede generarse como una extensión de una agrupación ya existente, que utiliza la red de redes para comunicarse entre sí. De igual forma, muchas otras comunidades virtuales pueden establecer juntas presenciales. Incluso muchos miembros interactúan en ambos ambientes. De esta forma, no es posible establecer que la virtualidad total sea un elemento esencial de una comunidad en línea, porque los miembros pueden moverse libremente de un medio a otro. Ni la virtualidad ni la presencialidad determinan la naturaleza de una comunidad en línea, sino más bien los lazos que puedan crearse en estos espacios (Kendall 2003: 1455).

El presente estudio de caso se centra en un portal argentino llamado Taringa!, que está ubicado en Buenos Aires, pero que sus miembros trascienden las fronteras provinciales y estatales creando una verdadera comunidad internacional. Nuestro objetivo es encontrar algunos aspectos dentro del discurso de los usuarios, que nos permita conocer hasta qué grado esta comunidad virtual a duplicado las instituciones del mundo material, y hasta qué punto los usuarios se consideran a sí mismos como miembros de una comuna. 


\section{La comunidad virtual Taringa!}

Ilustración 1

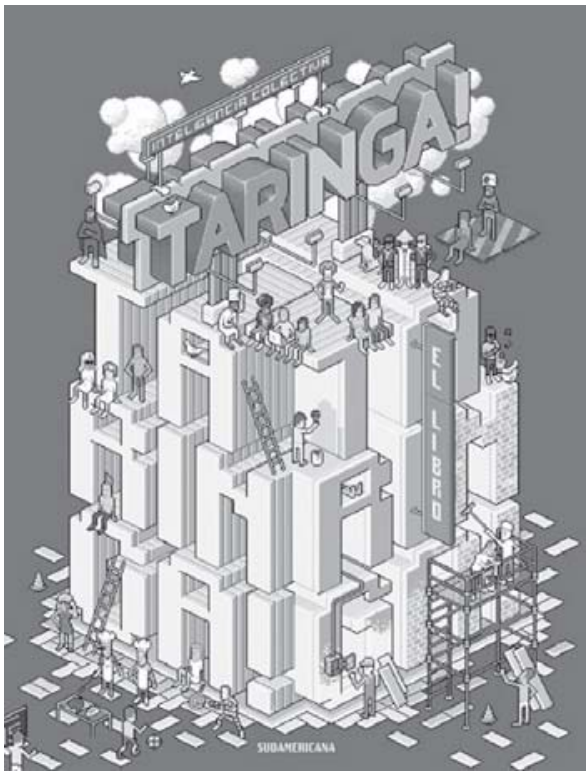

Portada. Libro Taringa! Ed. Sudamericana

Taringa! (www.Taringa!.net), es una comunidad de cibernautas que comparten información, desde ligas para descarga de música y películas, hasta monografías y ensayos. La mayor parte del contenido del sitio se encuentra apenas en la frontera de la legalidad: sobre todo por la violación de los derechos de propiedad intelectual. Los usuarios del sitio, sin embargo, denominan a las aportaciones "inteligencia colectiva”. Para los propietarios del portal, Matías Botbol, su hermano Hernán y Alberto Nakayama «La suma de las partes hacen un mejor sitio" (Faber y Bilbao 2009, secc. Sociedad).

Taringa! trabaja con apenas 12 empleados en unas pequeñas oficinas ubicadas en la Avenida Santa Fe, en Buenos Aires Argentina, y una treintena de moderadores que trabajan desde diferentes sitios del mundo, sin percibir salario alguno. Sin embargo, el sitio no es de ninguna forma pequeño: se reciben 2 millones de visitas, y se acrecienta con más de 5 mil posts (aportaciones) diarias, contando con cerca de 20 millones de usuarios registrados provenientes de todo el mundo. En palabras de unos de sus fundadores «Sólo hago lo que me gusta y tengo la suerte de tener un montón de gente que también le gusta lo que hago» (Gentinetti2010) 
La historia de Taringa! comienza, en el 2004, cuando fue creada por Fernando Sanz, para posteriormente ser adquirida en el 2006 por Alberto Nakayama y los hermanos Botbol (Matías y Hernán). Al momento de ser adquirida por sus actuales dueños, el portal contaba con 30 mil visitantes, con el paso del tiempo, y agracias a posteriores actualizaciones el sitio ha crecido de manera permanente «Teníamos la semilla del árbol, había que regarla» (Ibid), comenta Matías, uno de los dueños. No es muy claro de dónde surgió el nombre de Taringa!, sin embargo, existen dos versiones: Según la primera, el nombre de Taringa! nace en un zoológico, un simio hizo un ruido similar a Tah-inga, al sonar gracioso y llamativo se decidió poner dicho nombre. De acuerdo a la segunda versión, su nombre obedece a una ciudad Australiana Taringa! cuyo nombre en idioma aborigen significa: tarau (piedras) y nga (formado por).

Taringa! es uno de los sitios electrónico más visitados de Argentina, y también de América Latina. Su crecimiento, a partir del 2006, ha sido exponencial: En septiembre de 2008,el portal llegó contar con el usuario número 1.000.000 y 8 meses después, el sitio duplicó esa cantidad. El 2008 se considera que ha sido el mejor año de este portal: donde quedó un poco debajo de Google Argentina (google.com.ar) como el sitio más visitado de Argentina, superando las visitas a la versión digital del Diario el Clarín de Buenos Aires.

\section{Ilustración 2}

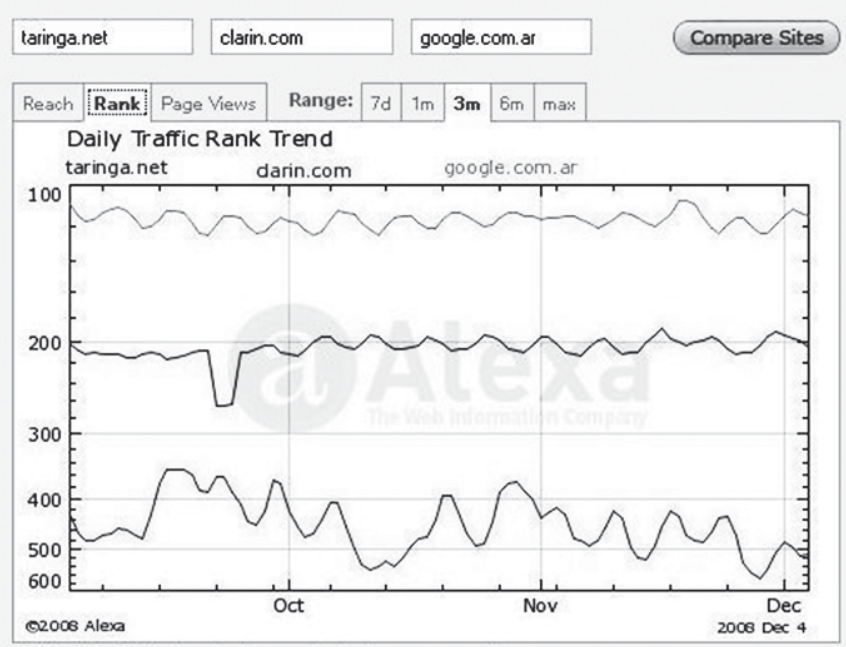

Get traffic details for: taringa.net clarin.com google.com.ar

Fuente Alexa (alexa.com) 
Si bien actualmente su afluencia ha disminuido, continúa siendo un espacio virtual muy concurrido. Para Octubre 2012, cuenta ya con 20, 004, 255 millones de usuarios registrados, un total de 15, 041, 129 aportaciones y con alrededor de 96, 814, 044 millones de comentarios sobre las aportaciones.

\section{El funcionamiento de la comunidad}

En Taringa! es posible compartir una amplia gama de contenidos mediante post (aportaciones). El sitio funciona de manera colaborativa, desde el usuario con más antigüedad como aquellos que inician, trabajando a través de aportaciones y comentarios sobre su calidad. No se permite la publicación de material pornográfico, para tal fin se creó un portal idéntico denominado Poringa (http://www.poringa.net), mediante el cual es posible compartir esta clase de contenidos. Aunque Poringa sea un sitio separado, tanto usuarios como moderadores de contenidos son los mismos que en Taringa! (“Henri” 2009).

Los usuarios registrados comparten noticias, ensayos, ligas para descargar videos, programas computacionales, música, entre otros materiales a través de posts. En estos aportes, es posible expresar comentarios sobre la calidad de la información compartida y en caso de aprobación, se les otorgan puntos mediante un sistema de calificaciones que le permite a cada usuario registrado, de acuerdo a su rango, entregar una determinada cantidad de puntos por día. Con base en estas puntuaciones se elabora una escala o ranking semanal de post y de usuarios, que muestra aquellos que recibieron más puntos. Con el tiempo los usuarios con más puntaje llegan a convertirse en moderadores y en algunos casos líderes de opinión dentro de la comunidad, lo cual nos lleva pensar en la existencia de una escala social vertical que se basa en la experiencia, pero también en la meritocracia, reflejada a través de los puntos ganados.

\section{Miembros de la Comuna: Los taringueros}

Establecer un perfil único para una comunidad internacional como Taringa! es una tarea imposible, sin embargo en palabras de los hermanos Botbol, un taringuero (Kukso 2008) "Es un hombre de unos 25 años que se mete en Internet sabiendo lo que es, que usa sin miedo el mail y el chat. Tiene un conocimiento avanzado, sabe cómo descargar música y está familiarizado con estas tecnologías. Las mujeres no están excluidas. Es más, su participación atraviesa un pleno crecimiento, aunque por ahora representan el 15\% frente al 85\% masculino. No se meten tanto con la computadora para descargar archivos como lo hacen los hombres. Las chicas son más de chatear, escribir mails y tener fotolog 9 ". El entusiasmo por pertenecer a la comunidad se puede apreciar de manera clara en la opinión de un usuarios, hablando sobre lo que significa ser un taringuero"Es una comunidad en la que te 
sentís como un amigo” (Gonzalo 2009) . Todos los usuarios, menos aquellos denominados novatos, poseen una cantidad de puntos para poder evaluar los post de sus compañeros. Cada vez que se vota, el autor del aporte va ganando puntos y aumentado en su ranking. Los rangos o tipos de usuarios son ${ }^{10}$ :

Administrador: poseen los mismos privilegios que los Moderadores pero además, están encargados del constante desarrollo del sitio en materia técnica.

Moderador: un moderador es el encargado de mantener el orden, la paz y el respeto en Taringa!, prestando atención a las políticas de privacidad. Cuenta con 35 puntos por día.

Gold User: usuarios que se posicionan entre el puesto 1 y 50 del T! Rank $^{11}$. Diariamente pueden otorgar 30 puntos.

Silver User: usuarios que se posicionan entre el puesto 51 y 100 del T! Rank. Diariamente pueden otorgar 20 puntos.

Great User: es un premio para aquellos usuarios que lo merecen, no hay criterios específicos para su otorgamiento y la única diferencia con los New Full Users o los Full Users, es que poseen 17 puntos por día.

Full User: poseen los mismos privilegios que los New Full Users. Se denomina Full Users a quienes se registraron antes de la versión 3 de Taringa! (Marzo de 2007) o a quienes fueron en alguna oportunidad Silver User o Gold User y dejaron de serlo por salir del top 100 de usuarios. Estos miembros pueden otorgar 12 puntos por día.

New Full User: luego de obtener 50 puntos en un solo posteo, los Novatos se convierten en New Full Usersy pueden hacer uso pleno de Taringa!, incluidos los comentarios y posteos en la sección general. Poseen 10 puntos por día para calificar a otros posts.

Novatos: son los Usuarios recién llegados a la comunidad. Su actividad está restringida a postear y comentar en la sección Novatos, pero su acceso al contenido del sitio es total. No pueden dar puntos.

El sistema de ranking privilegia no sólo el compartir un recurso, hay miles de posts que no cuentan con puntos, sino que los propios miembros determinan el valor de cada aporte. Sin embargo, hay miembros de la comuna que tienen un mayor peso (pueden aportar más puntos), que otros. Cuando un aporte es recibido con aceptación por un gran número de usuarios, el autor puede ser catapultado hacia niveles de mayor importancia dentro de la comunidad. Este tipo de movilidad social tiene un símil con el de las sociedades de cazadores, donde el cazador más experimentado que logra las mejores presas, puede aspirar al mayor reconocimiento dentro del núcleo social. 


\section{El control}

"Desconocemos si el contenido de un post tiene derechos», responden, casi de manual. Y aclaran: «Siempre que nos llegue una denuncia del titular de esos derechos, eliminamos el post”12

La estructura del sitio Taringa! se basa en las aportaciones de los usuarios, pero generalmente se trata de contenido redactado por terceros. En este sentido los usuarios no con productores, no se trata de una comunidad agricultora o ganadera sino más bien de recolectores o cazadores. Pero cuidado, llegar a destacar en una sociedad dedicada a la recolección o la caza (En este caso de información) no es tarea fácil. No se trata de robarse lo que otros han recolectado antes. En caso de que un post creado por un usuario, fuese una copia exacta de otro ya publicado en la propia comunidad o en otra, sin que se reconociese la autoría original, un moderador procedería a borrarlo. Ciertamente el moderador no sabe al principio distinguir la originalidad de la aportación, pero los mismo usuarios se encargar de imputar el cargo.

Como el caso de Taly Online(Taly 2008)una usuaria argentina que sufrió el robo de una aportación de su autoría publicada en su Blog personal y que fue compartida en Taringa! a título personal de un usuario. Después de una denuncia de la autora se procedió a la eliminación del aporte. Para los usuarios de la comunidad una cosa es compartir información de terceros, y otra muy distinta el robo de la autoría. El prestigio en esta clase de comunas se basa siempre en la confianza y la aprobación, cuando existe el fraude, el infractor es castigado, porque se considera una afrenta a la comunidad (Marccur 2008).

Los autores de las faltas reciben un apercibimiento, porque se considera que al ser una comunidad que vive del intercambio de buena fe, se trató de un error causado por la ignorancia. El aporte en cuestión es eliminado, pero si se reitera en esta conducta puede suspenderse temporal o permanentemente la cuenta del usuario, según la gravedad del caso.

El moderador de contenidos es la policía que se encarga de revisar que los aportes cumplan con las políticas de uso del portal ${ }^{13}$. Su tarea no sólo implica el apercibir usuarios infractores y borrar contenidos, sino que también puede recordar de vez en cuando las normas que rigen a la comunidad. Como en el caso del moderador Medulac ${ }^{14}$.

\section{"Para recordarles, de paso lo vuelven a leer:}

De los post:

Se eliminan los post que contengan:

- Chistes escritos, adivinanzas, trivias.

- Cosas morbosas tales como cadáveres, vómitos, violaciones, sangre, heridas, enfermedades, etc.

- Información personal propia o de terceros tales como e-mails, msn, nombres, teléfonos, etc. 
- Material que ya fue posteado anteriormente (famosos repost). Excepto descargas que pueden dejar de funcionar con el tiempo.

- Noticias que no contienen fuente. (Es necesario agregar el link correspondiente).

- Títulos poco descriptivos, que sean CON MAYUSCULA (parcialmente o totalmente) o $\{\{\{$ QuEQUiErAnLLamaR la AtenCiÓN!!! \}\}).

- Mujeres demasiado provocativas, en bikini, semi-desnudas o estén en situaciones que puedan ser consideradas sexuales.

- Contenido de mala calidad o mal hecho.

- Links a páginas/blogs personales/propias/amigos (SPAM).

- Mensajes a otros usuarios o a los moderadores.

- Mensajes o contenido con el claro objetivo de buscar polémica. (política, fútbol, etc.).

- Contenido que se relacione o haga \»apología de delito\» (venta de droga,violencia,delincuencia). La violencia es un delito.

- Links de Torrents,Pando, emule, etc. (P2P, P2M).

- Passwords o accesos privados (Rapidshare, Megaupload, cuentas de correo, etc.).

- Sorteos no autorizados por un moderador. No pueden ser realizados por novatos.

Se cierran los post que contienen:

- Temas demasiados políticos que pueden llegar a ofender a otros usuarios, ya sea por su contenido o comentarios.

- Post que se transforman en un foro o se genera polémica. (famoso forobardo)."

La anterior transcripción demuestra la imposición de patrones morales muy específicos, pero también aspectos técnicos sobre el tipo de post a utilizar. Al tratarse de un sitio argentino, los temas sobre futbol y política están totalmente excluidos.Por el contrario, una prohibición casi general en Internet, un asunto de nettiqueta, es la no utilización de letras en mayúscula, porque son sinónimos de que seestá gritando dentro de la comunidad. Al analizar esta clase de advertencias es muy claro como reflejan cuestiones meramente técnicas, otras de carácter interno de la comunidad, pero otras son eminentemente legales, es decir pertenecientesa cuerpos normativos estatales.

\section{Taringa! y los bemoles de las comunidades virtuales}

Una comunidad virtual puede albergar desde unas decenas hasta millones de miembros, pero quizá más asombroso que la cantidad, sea la forma en que se pueden establecer pautas de comportamiento bien definidas entre los usuarios de estos espacios. En todas ellas hay roles bien definidos que cada individuo, sin importar su procedencia termina por acatar, casi por lo general sin ningún contratiempo. Un elemento esencial de toda comunidad es este cumplimiento puntual de roles, cuya función y objetivos 
han sido definidos prácticamente desde la creación de la comunidad misma, y su permanencia depende de su respeto.

Pero toda comunidad pervive únicamente como una extensión del mundo físico, y Taringa! no puede ser explicada sin este referente. Por que cada tema que se abre y cada comentario que se plasma lleva la impronta de los sentimientos, las preocupaciones y los anhelos de un mundo físico desde cual se construye el virtual. Las comunidades en línea son complementos de las esferas sociales de los individuos, y su objetivo es solventar las mismas necesidades que se puedan tener en los espacios de interrelación social de los miembros: desde la búsqueda de relaciones amorosas hasta las actividades mercantiles. De ahí que no es posible argüir que una comunidad virtual no pueda ser considerada como tal por el simple hecho de no existir de manera física. Lo que crea, en sentido sociológico, una comunidad no subyace en el formato, natural o virtual, sino más bien en la intencionalidad y la intensidad con que los individuos buscan expandir su esfera de relaciones sociales.

Los espacios de intercambio en línea como Taringa! recaen en el concepto de comunidades no sólo por la cuestión del compromiso de sus miembros, sino también porque al momento de ser creadas, sus fundadores vertieron en ellas las mismas instituciones con las que se desenvuelven en el mundo "off-line" y son precisamente las normas emanadas de estas instituciones las que han permitido que estos espacios se consoliden y subsistan en el tiempo.

\section{Conclusión}

Las comunidades virtuales representan una ampliación de las relaciones sociales tradicionales. Los motivos para crear una comunidad son variados: desde el simple intercambio de material, hasta la creación de debates y grupos de discusión. Todas las comunidades utilizan ineludiblemente medios de control y represión, porque sólo de esta manera es posible formalizar y asegurar el funcionamiento del espacio colectivo. Al hacer uso de mecanismos tradicionales represivos,es imposible crear comunidades digitales con carácter utópico o que estén fuera de estándarescomúnmente aceptados.

El análisis del portal electrónico de Taringa!demuestra claramente como el proceso de control de las acciones de los miembros se va interiorizando poco a poco en la comunidad, y llega a institucionalizarse de tal manera que no es necesario el uso de la fuerza coercitiva a través de actos patentes de autoridad, sin embargo, de vez en cuando la policía (moderadores) deben de hacer manifiesto ese control a través advertencias, imputaciones y castigos.

La consolidación del ciberespacio en la vida cotidiana ha venido a potencializar la creación, mejoramiento o consolidación de comunidades 
virtuales, sin embargo, este hecho por sí, no demuestra un cambio de paradigma en la forma en que las personas se han venido relacionando unas con otras, sólo demuestra la existencia de un nuevo espacio desde el cual es posible ampliar las posibilidades sociales existentes, un medio para asentar, intercambiar o modificar instituciones culturales. 


\section{Notas}

${ }^{1}$ En la filosofía platónica es el mundo de las ideas, el lugar donde se encontraban las formas perfectas de las cosas. El hombre en su vida cotidiana sólo podía apreciar una imagen parcial y borrosa de la realidad. Dicha imagen era un reflejo de este topusuranos, o mundo de las ideas.

${ }^{2}$ Muestras de la utilización de metáforas en la definición de Internet, pueden verse con mayor claridad en las siguientes normativas estadounidenses: US Congress (1996) The Communication Decency Act. US Congress (1998) The Children's Online Protection Act. Ambas declaradas anticonstitucionales por la Suprema Corte de Justicia de Estados Unidos.

${ }^{3}$ Por modelos represivos no se hace alusión no aun gobierno tiránico o despótico, tampoco a regímenes totalitarios, sino más bien al hecho inherente de que todo marco normativo formal o informal, es inminentemente coercitivo, porque busca ante todo moldear la conducta y establecer pautas de comportamiento que modulen la actividad de los individuos. En este sentido, no hay ciudad ni comunidad que carezca de estos marcos, pero no quiere decir que las normas impuestas sean moralmente buenas o malas.

${ }^{4}$ Ejemplo de un nombre de dominio del sitio del Colegio de Jalisco. Su dominio es coljal.edu.mx.

${ }^{5}$ Los sitios de Internet que por su naturaleza necesitan estar permanentemente conectados, generalmente tienen una dirección IP que los identifica, permitiendo el intercambio con otros sitios.

${ }^{6}$ La palabra backbone, se refiere a las principales conexiones troncales de Internet. Está compuesta de un gran número de routers comerciales, gubernamentales, universitarios y otros de gran capacidad, que se encuentran interconectados y que llevan los datos a través de países, continentes y océanos del mundo.

${ }^{7}$ SecondLife es una plataforma desarrollada por la empresa Linden Research, que permite a los usuarios interactuar entre sí en un mundo virtual.

${ }^{8}$ Como ya se ha mencionado anteriormente, tanto las comunidades virtuales como sus normas son adecuaciones del mundo físico.

${ }^{9}$ Bitácora personal con fotografías.

${ }^{10}$ La siguiente información está tomada del post ¿Como se llaman todos los rangos de Taringa! ? en el espacio de T! Respuestas (http://www.Taringa!.net/comunidades/ Taringa!respuestas/292151/\%C2\%BF-Como-se-llaman-todos-los-rangos-de-Taringa!-.html)

${ }^{11}$ El T! Rank (Taringa! Rank), es un ranking de usuarios interno, que se genera diariamente gracias a una fórmula matemática que calcula la participación y el contenido que los usuarios comparten en el sitio. A los primeros 50 usuarios del ranking se los cataloga como Gold Users, los cuales pueden otorgar hasta 30 puntos diarios a otros mensajes. Los usuarios posicionados entre el puesto 51 y 100 se los cataloga como SilverUsers, pudiendo otorgar hasta 20 puntos diarios. Cuando un usuario deja de estar entre los primeros 100 del ranking se les recataloga como Full Users, pudiendo otorgar hasta 12 puntos diarios. Fuente Wikipedia 
(http://es.wikipedia.org/wiki/Taringa!\#T.21_Rank)

${ }^{12}$ Extracto de la entrevista a los dueños del portal Taringa! En Farber y Bilbao, 2008.

${ }^{13}$ Taringa! Cuenta con un extenso documento sobre las políticas de uso del sitio (http:// www.Taringa!.net/terminos-y-condiciones/) donde se explica, además de otras cuestiones, el tipo de aportes permitidos. Así por ejemplo en el apartado 8.1 Señala:

Se considerará como Uso prohibido, entre otros, la fijación de post, mensajes o comentarios, propaganda, así como la indicación de vínculos a páginas Web, que:

1. Resulten ofensivos para los derechos personalísimos de los individuos, con especial referencia al derecho al honor, a la dignidad, a la intimidad, a no ser objeto de tratos discriminatorios, a la salud, a la imagen, y a la libre expresión de las ideas, con absoluta independencia del cuerpo legal donde tales derechos adquieran reconocimiento.

2. Infrinjan los derechos de propiedad intelectual de terceros.

3. Posea contenido inapropiado.

4. Tenga por objeto vulnerar la seguridad, y/o normal funcionamiento de los sistemas informáticos de Taringa! o de terceros.

5. Induzca, instigue o promueva acciones delictivas, ilícitas, disfuncionales o moralmente reprochables, o constituya una violación de derechos de propiedad intelectual de terceras personas.

6. Incorporen alguna forma de publicidad o fin comercial no permitidos por Taringa!. 7. Tenga por objeto recolectar información de terceros con la finalidad de remitirles publicidad o propaganda de cualquier tipo o especie, sin que ésta fuera expresamente solicitada.

${ }^{14}$ A continuación se transcribe el texto íntegro del moderador, sin cambiar el color ni la redacción del texto. Este documento es importante porque nos permite ver la imposición de una moral particular para el tipo de contenidos de la colectividad. En este sentido las formas de conductas de lostaringuerosse tienen que acoplar a lineamientos poco claros como por ejemplo morboso o bien Mal hecho. Sin embargo, por los comentarios de los usuarios, se nota claramente que hay un entendimiento, una interiorización y por lo tanto una aceptación de estos parámetros que desde fuera pueden parecer ambiguos. Para consultar el documento íntegro se puede ingresar a la siguiente liga (http://www.Taringa!.net/posts/Taringa!/ 1717237/Sobre-las-fuentes-en-Taringa!_html) 


\section{Bibliografía}

Adams, Tyrone (comp),(2008), Electronic Tribes: The Virtual Worlds of Geeks, Gamers, Shamans, and Scammers, University of Texas Press, Austin.

Anderson, Benedict R (1991), Imagined Communities: Reflections on the Origin and Spread of Nationalism, Verso, London.

Bell, David (2001), An Introduction to Cybercultures, Routledge, London.

Boyer, M. Christine (1996), CyberCities: Visual Perception in the Age of Electronic Communication, Princeton Architectural Press, New York.

Burk, Dan, L (1999), Cyberlaw and the Norms of Science, Intellectual Property and Technology Forum, Boston.

Castells, Manuel (2004), The Power of Identity, Blackwell, Malden Mass.

Farber, Maria y Bilbao, Horacio (29 de enero de 2009), La polémica e imparable comunidad de Taringa! es un éxito en Internet, El Clarín, Recuperado dehttp://www.clarin.com/diario/2009/01/30/um/m-01848906.htm

Fernback, Jan y Thompson, Brad (Mayo de 1995), Computer-Mediated Communication and the American Collectivity: The Dimensions of Community Within Cyberspace, Annual convention of the International Communication Association, Convenciónanualllevada a cabo en Albuquerque, New Mexico.

Fuchs, Christian (2008), Internet and Society: Social Theory in the Internet Age,Routledge, New York.

Gentinetti, Mauro (5 de Agosto de 2010), Taringa!: el éxito de internet convocó a cientos de jóvenesDiario la Opinión. Recuperado http:// www.laopinion-rafaela.com.ar/opinion/2010/08/05/c080590.php

Geertz, Clifford (1973), The Interpretation Of Cultures, Basic Books, New York.

Giannachi, Gabriela (2004), Virtual Theatres: An Introduction, Routledge, London.

Gibson, Wiliam (1989), Neuromante, Ediciones Minotauro, Barcelona. Gonzalo (Usuario) (2009), Másallá de la Red Taringa!Recuperado en http:/ /www.losandes.com.ar/notas/2008/7/2/estilo-367261.asp

Grosz, E. A. (2001), Architecture from the Outside: Essays on Virtual and Real Space, MIT Press, Cambridge Mass.

“Herni” (Moderador) (2009), Taringa! - Sobre el presente y futuro de 
Taringa!, Recuperado dehttp:/www.Taringa!.net/posts/Taringa!/2404878/ Sobre-el-presente-y-futuro-de-Taringa!.html

Kendall, Lori (2003), «Virtual Communities», En Encyclopedia of Community: From the Village to the Virtual World (p. 1455), Sage Publications, Thousand Oaks.

Kukso, Federico (9 de marzo de 2008), Una comunidad virtual reargenta,Crítica de la Argentina. Recuperado de http:// www.criticadigital.com/impresa/index.php?secc=nota \&nid=531

Lessig, Lawrence (2001), El código y otras leyes del Ciberespacio,Taurus, Madrid.

Luckmann, Thomas y Berger, Peter (1995), La construcción social de la realidad, Amorrortu, Buenos Aires.

Maccur (10 de julio de 2008), Taringa! responde a las críticas y pide disculpas por el accionar de sus usuarios, Recuperado de http:// www.puntogeek.com/2008/07/10/Taringa!-responde-a-las-criticas-y-pidedisculpas-por-el-accionar-de-sus-usuarios/

Matías, Paúl (1998), La ciudadInternet, Bellaterra, España.

Palloff, Rena M. y Pratt, Keith (2007), Building Online Learning Communities: Effective Strategies for the Virtual Classroom, (2 ${ }^{\mathrm{a}}$ ed.), Jossey-Bass, San Francisco.

Rheingold, Howard (1993), The Virtual Community: Homesteading on the Electronic Frontier, Addison-Wesley, Massachusetts.

Taly (2008), Crónica de una denuncia agitada, Recuperado de http:// www.taly.com.ar/blog/cronica-de-una-denuncia-agitada/

Recibido: 24.04.2012

Aceptado: 07.11.2012 